\title{
Mathematical Model of the Distribution of Laser Pulse Energy
}

\author{
Pavels Narica Artis Teilans Lyubomir Lazov Pavels Cacivkins Edmunds Teirumnieks \\ Faculty of Engineering, Rezekne Academy of Technologies, Latvia, \\ lasererta.lv
}

\begin{abstract}
Method allows for modelling of the complex process of laser pulse energy distribution over flat work surface. The process of calculating the correct result does not use common lasing formulas but instead employs the mathematical model of matrix multiplication of three input matrices representing a pulse model, a line model, and a plane model. The pulse model represents the distribution of planar energy densities within the laser pulse. The line model represents the distribution of pulses within the line. The plane model represents the distribution of lines within the plane. Because mathematical model is implemented within a spreadsheet processor, its size can be adjusted as needed and it can be instantiated multiple times for simultaneous modelling of different input parameters.
\end{abstract}

Keywords: mathematical model, modelling method, laser pulse, energy distribution, planar energy density, matrix multiplication, laser marking, spreadsheet processor

\section{Introduction}

The main goal of this research is to simplify the process of understanding and visualizing the distribution of laser pulse planar energy densities over flat work surface.

Laser systems are widely used and provide some spectacular capabilities in many different fields. However lasers are complicated systems and operating a laser system requires a very good understanding of how lasing is actually done and how it can affect surroundings.

In scientific literature and on the internet there is a plenty of information about the laser systems. Such information contributes to better understanding of the lasing processes and includes some of the most widely used formulas and concepts, such as distance between two consecutive pulses, distance between two consecutive lines of pulses, individual laser pulse energy, and average lasing power (Bliedtner et al, 2013). However, the problem with such formulas and concepts is that they do not necessarily help one visualize the process being calculated. Another issue is that lasing processes are expensive.
Laser system usually provides for its operator a set of technical parameters which can be adjusted to obtain the necessary results. Nevertheless, these technical parameters may not provide a clear understanding of how they affect the lasing results. Even though laser system's operator might use provided technical parameters in formulas, the results of such formulas per se do not ensure being sufficiently useful.

In practice there are two main types of laser systems - pulsed lasers and continuous-wave (CW) lasers (Eichler, 1998). The former deliver energy to the work surface in discrete packets called pulses, while the latter emit photons continuously. Many of commonly used formulas are better suited for $\mathrm{CW}$ type laser systems, as these laser systems produce more predictable results. When such formulas are used in relation to pulsed laser systems, the results of formulas usually contain averaged values without local minimums and maximums.

Local minimums and maximums occur during both pulsed and CW type lasing and play an important role on produced results, as regions of work surface that are exposed to higher planar energy densities would behave differently than other remaining regions (Laakso et al, 2009; Antonczak et al, 2014). This is especially the case when producing colour laser marking on metals, as formed thin oxide films differ in regions exposed to higher planar energy densities compared to those exposed to lower planar energy densities, and thus have different appearance which has its contribution to overall perceived colour of marking (Ming et al, 2008; Veiko et al, 2014).

Pulsed laser systems emit pulses with some pulse repetition rate in the direction of scanning. Pulse repetition rate, scanning speed, the direction of scanning, pulse width, average lasing power, distance between two consecutive lines, and many other technical parameters are all set by laser operator. Because there are time periods between each two consecutive laser pulses, when no additional energy is delivered to the work surface, each two consecutive pulses may overlap in many different ways and thus distribute their energy over the work surface in many different forms. Each such distinct form of energy distribution in the end can affect work surface differently. 
As already stated above, commonly used formulas do not allow for clear and instant understanding of how distribution of laser emitted energy over flat work surface would look like in practice and where its local maximums and minimums would be located. The best results common formulas can help to achieve, when given values of main technical parameters are known and laser construction specifics are taken into account, are to find distances between each two consecutive pulses and each two consecutive parallel lines of pulses as well as energy content of individual laser pulse and average lasing power that represents the rate of laser emitted energy delivery to the work surface.

When one knows the total amount of laser pulses delivered to a unit area of work surface as well as the energy content of each individual pulse, one may further calculate average planar energy density for that unit area. However, just by using common formulas it would not be easy to obtain information about the actual distribution of different planar energy densities, as these can differ based on their position on the work surface due to the overlapping effects between each two consecutive pulses and each two consecutive parallel lines of pulses.

The present mathematical model allows for modelling of laser pulse energy distribution over flat work surface. In particular, it is a novel method that deploys built-in spreadsheet processor's matrix multiplication function in order to automatically generate informative numeric data in form of a twodimensional histogram that can be further used for visualizing the actual distribution of laser pulse planar energy densities over flat work surface.

\section{Materials and methods}

Experiments were carried out using PowerLine F-20 Varia series pulsed fiber laser system produced by Rofin-Sinar Laser GmbH. It emits photons of wavelength equal to $1064 \mathrm{~nm}$, has maximum average lasing power of $20 \mathrm{~W}$, pulse repetition rate of 2-1000 $\mathrm{kHz}$, adjustable pulse width of 4-200 ns.

The colour palette shown in Figure 1 contains the yellow colour which is also called sample colour 4 throughout the text. The image in Figure 2 was taken using optical microscope Meiji Techno MT and represents the sample colour 4 . The stainless steel sample used for colour laser marking was 4301 18-9E $2 \mathrm{R}$.

The sample colour 4 described is Figure 1, Figure 2, Figure 3, and Figure 4 has the following technical laser parameters associated with it: pumping power of $25 \%$ (equivalent of $2 \mathrm{~W}$ average power, given specified pulse repetition rate and pulse width), pulse repetition rate of $200 \mathrm{kHz}$, scanning speed of $200 \mathrm{~mm} / \mathrm{s}$, pulse width of $4 \mathrm{~ns}$, and distance between two lines of $5 \mu \mathrm{m}$.

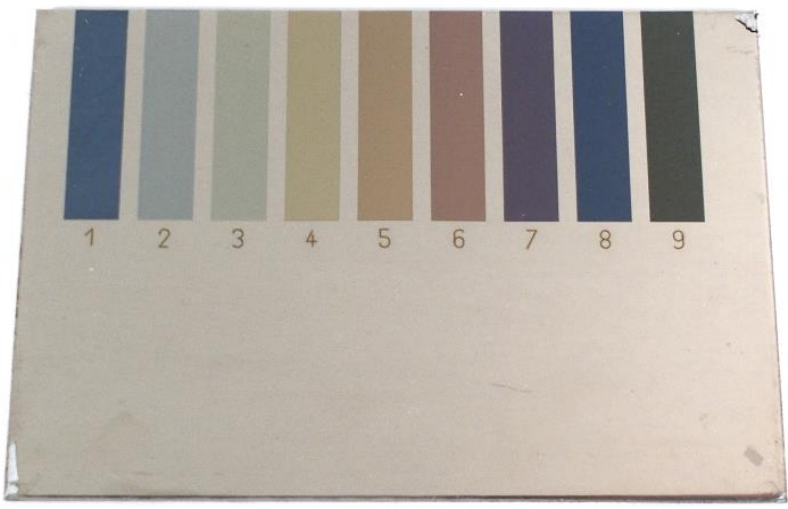

Figure 1. Produced sample marking colours on stainless steel workpiece.

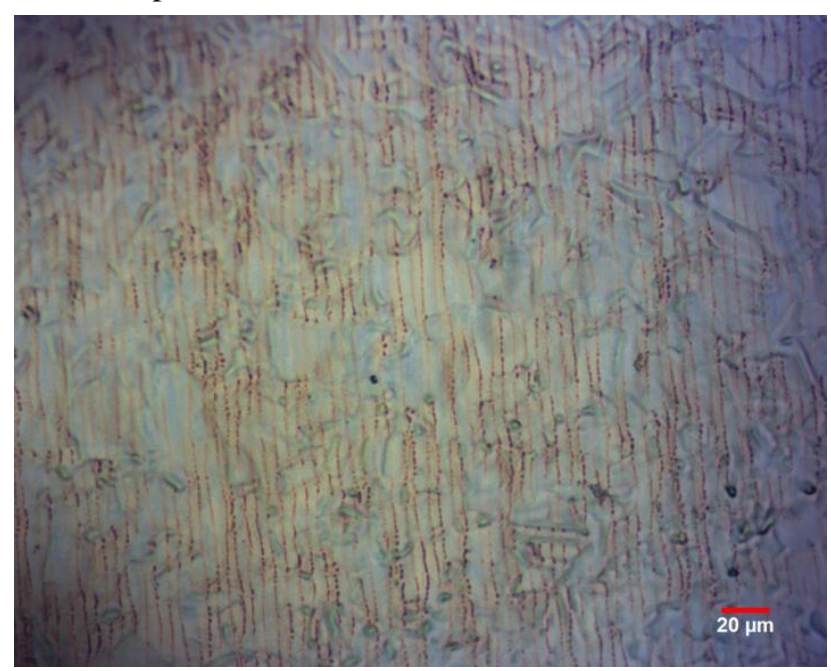

Figure 2. Sample marking colour 4 under optical microscope.

The method of modelling the distribution of laser pulse planar energy densities over flat surface allows for analysis of lasing processes by providing a mathematical model which consists of six related matrices. No common lasing formulas are used by the mathematical model, though any model's user can always derive necessary lasing formulas from the model by analyzing its state. The present method can provide different kinds of relevant information about laser pulse energy distribution over some flat surface, such as linear energy densities, linear pulse densities, distance between two consecutive pulses or lines of pulses, individual laser pulse energy.

The aim of the method is to make the most prominent feature of laser systems easier to understand, define, quantify, visualize, teach, and simulate by referencing it to existing and usually commonly accepted knowledge and formulas. The method offers a new way of looking at what actually happens with laser pulses, as they are being accumulated on some work surface. 

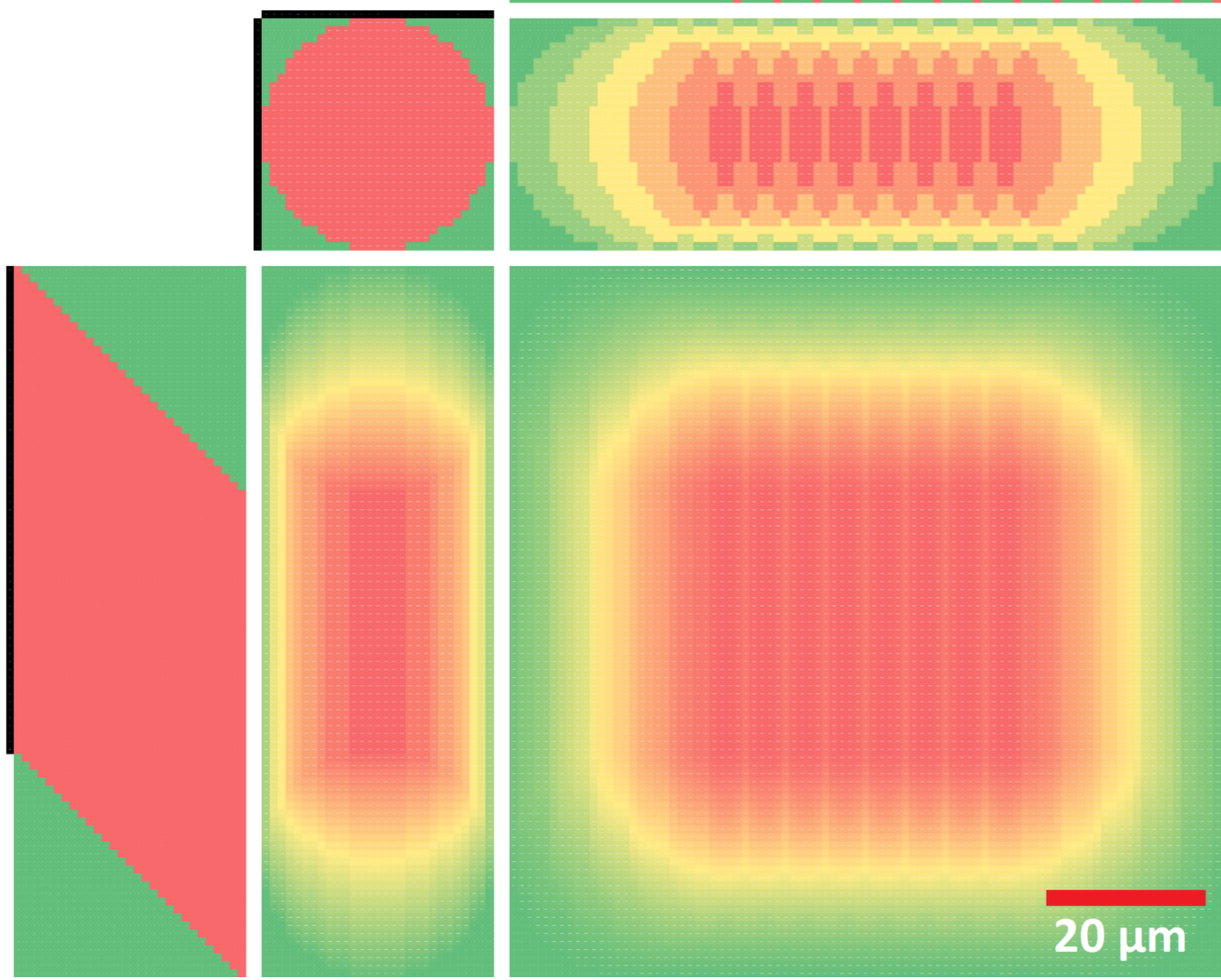

Figure 3. Produced visualization of the distribution of laser pulse planar energy densities for sample colour 4.

The method allows users to simulate actual distribution of planar energy densities over flat work surface. This is accomplished by interacting with model's input area consisting of numeric square matrix "P" (Figure 5), dedicated for defining distribution of planar energy densities within the pulse itself, as well as two perpendicular numeric vectors " $y$ " and " $x$ ", the former allowing to define distribution of pulses within a line and the latter allowing to define the distribution of these lines within a work surface plane. Numeric input of column vector " $y$ " is automatically copied into each remaining column vector of matrix "Y" and shifted along the length dimension of the matrix such that diagonal lines of identical numbers are formed. Numeric input of row vector " $\mathrm{x}$ " is automatically copied into each remaining row vector of matrix " $\mathrm{X}$ " (Figure 6) and shifted along the width dimension of the matrix such that diagonal lines of identical numbers are formed.

The most important aspect of the method is that it correctly simulates the actual distribution of planar energy densities over flat work surface from the aspect of both - physics and mathematics. By multiplying three matrices "Y", "P", and "X" the mathematical model is able to calculate positions of delivery of every laser pulse relative to the flat work surface. The distribution of laser pulses is immediately displayed back in form of three output matrices "YP", "PX", and "YPX". The numeric information of matrix "YPX" can easily be visualized as a three-dimensional surface 
chart to display the distribution of laser pulse planar energy densities (Figure 4).

\section{Laser pulse energy distribution}

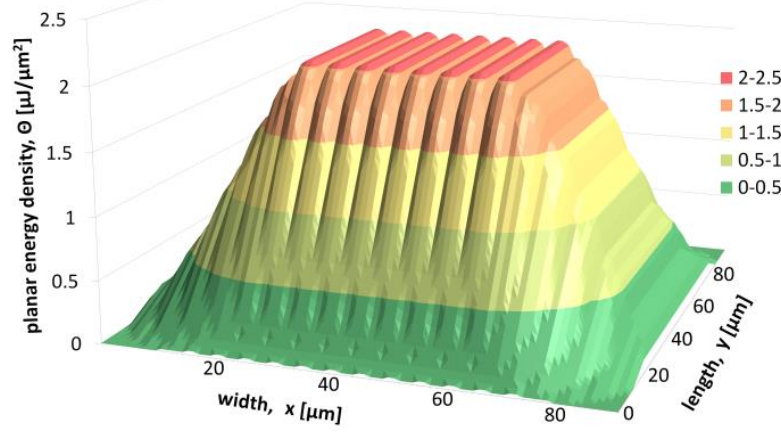

Figure 4. Three-dimensional surface chart of the distribution of laser pulse planar energy densities for sample colour 4 .

\section{Results and discussion}

The method of modelling the distribution of laser pulse planar energy densities by the use of matrix multiplication is implemented by the mathematical model comprised of six related matrices - three input matrices and three output matrices (Figure 7). The input matrices accept user provided data in form of numbers. The output matrices update their numeric states every time user makes changes to the input matrices.

The method always produces correct results because of its simple underlying logic that photons are both

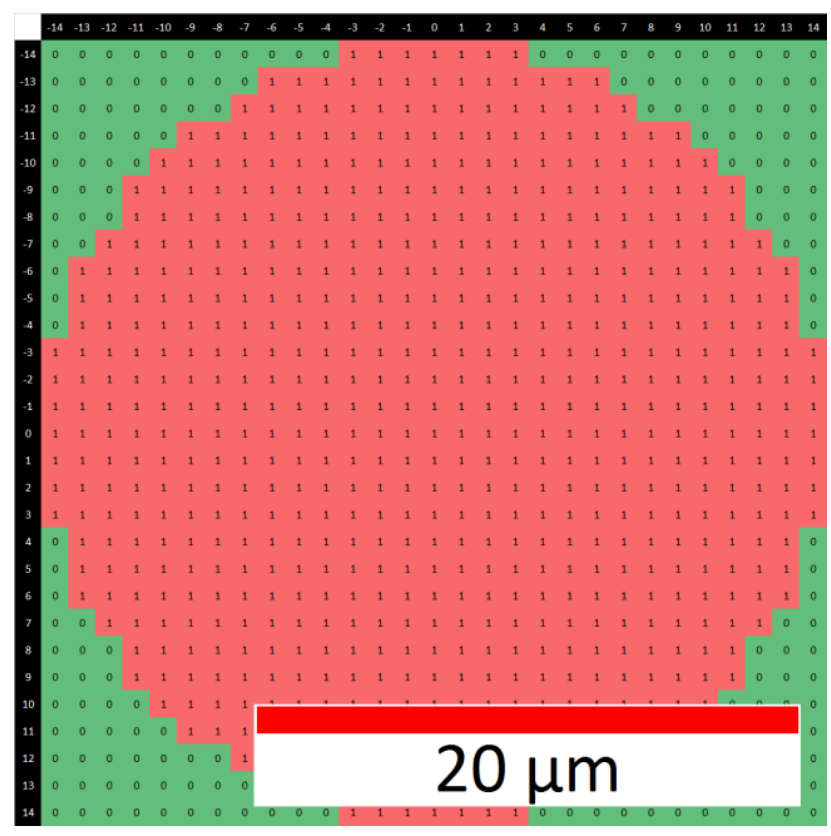

Figure 5. Example of pulse model "P".

carriers and units of energy and that they are additive. Thus laser pulse is a representation of some quantity of photons, and laser pulse total energy is always proportional to the amount of photons it consists of due to energy being an extensive physical property. The mathematical model stores one such laser pulse as a model itself in a form of a square matrix "P". It consists of numeric data that represent arbitrary amounts of photons and their relative positions within that laser pulse's planar surface projection. The mathematical model then uses this user defined or default laser pulse model to copy and to distribute it over the flat work surface.

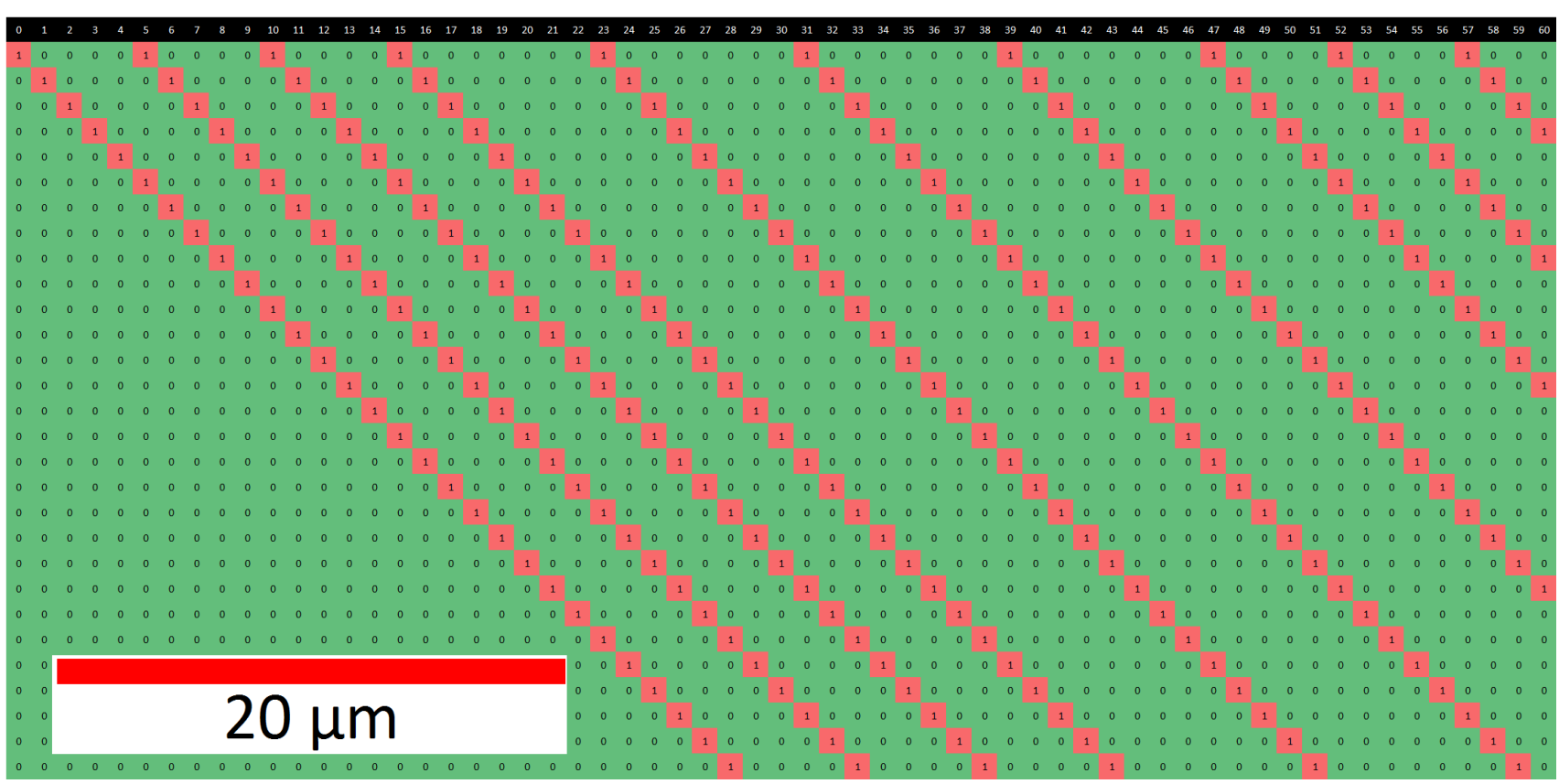

Figure 6. Example of line model "X". 


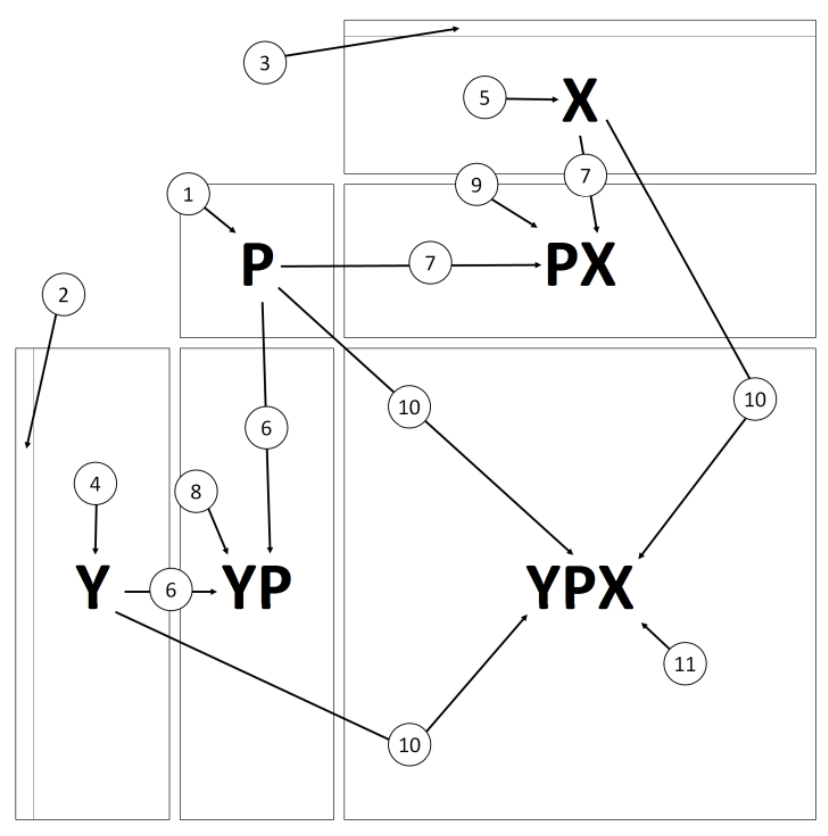

Figure 7. Schematic view of mathematical model implemented by the present method.

Besides input matrix " $\mathrm{P}$ " there are two perpendicular input matrices "Y" and " $\mathrm{X}$ " representing line model and plane model respectively. Both input matrices "Y" and " $\mathrm{X}$ " serve identical functions of storing positions of where laser pulses or lines of pulses are to be delivered in relation to the work surface. Input matrix "Y" is associated with flat work surface's length dimension, while input matrix " $\mathrm{X}$ " is associated with its width dimension. When viewed, the input matrix "Y" appears to have vertical rectangle shape with its width equal to the side length of square matrix "P", while the input matrix " $\mathrm{X}$ " appears to have horizontal rectangle shape with its length equal to the side width of square matrix "P". The work surface is itself represented by an output matrix "YPX" with its length equal to the length of matrix "Y" and its width equal to the width of matrix " $\mathrm{X}$ ".

The mathematical model calculates three different matrix products and stores them in the output matrices "YP", "PX", and "YPX" to display the distribution of laser pulse planar energy densities from three different points of view: as two perpendicular to one another laser pulse lines "YP" and "PX", and as a matrix representation of work surface "YPX".

Both input matrices "Y" and "X" are based on the idea of the identity matrix which is a special case of matrix in mathematics that does not change other matrices it is being multiplied with. Input matrices " $\mathrm{Y}$ " and " $\mathrm{X}$ " are not themselves identity matrices, yet they share some very important similarities - only diagonal lines of numbers are stored in them and each distinct diagonal line consists of identical numbers specified by mathematical model's user within the first column vector " $y$ " of input matrix "Y" and the first row vector "X" of input matrix "X".

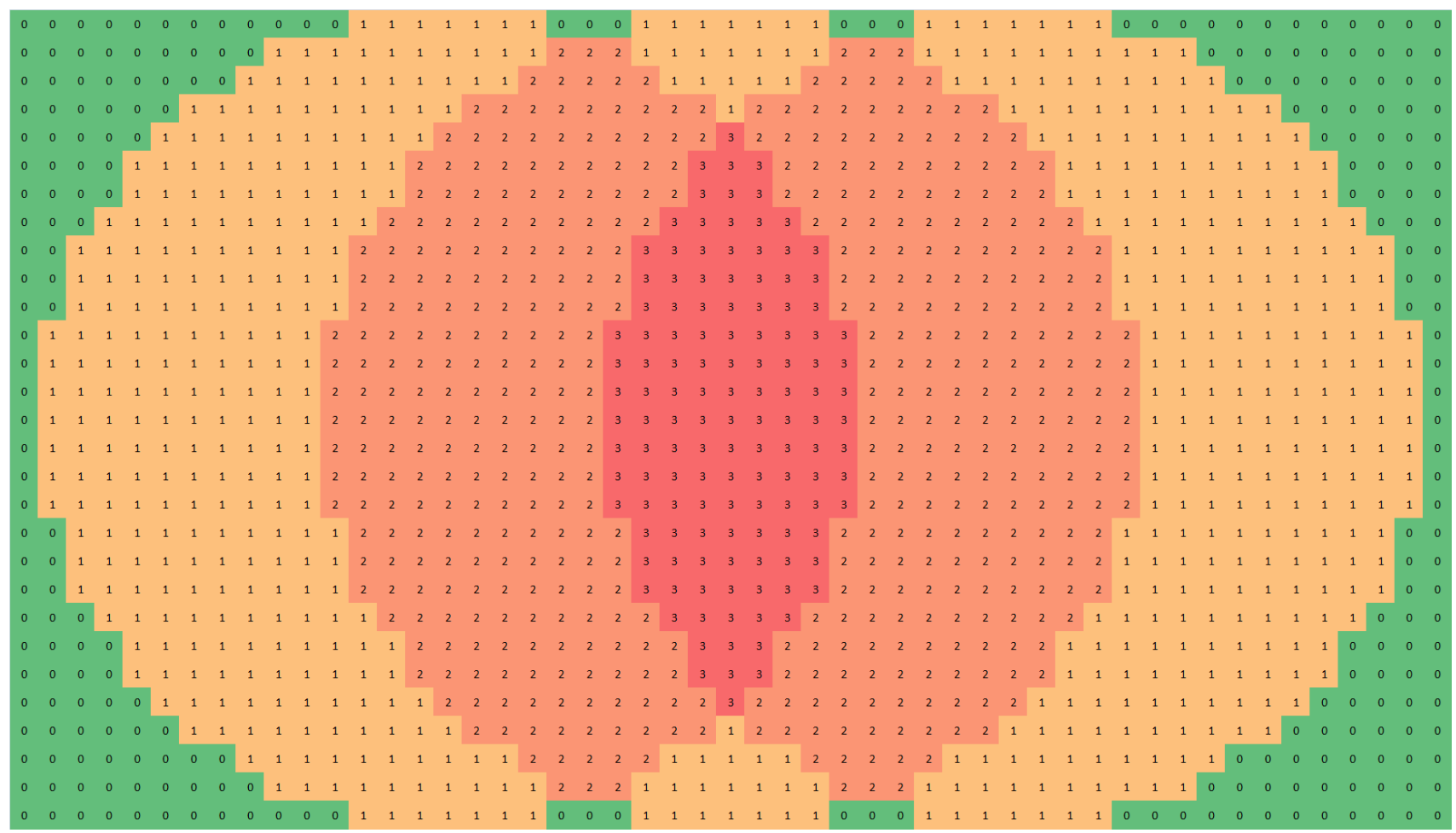

Figure 8. Example sum of three partly overlapping laser pulses. 
Such energy content addition is in accordance with the laws of physics and is provided by mentioned procedure of multiplication of input matrices. When three or more pulses are to be somehow overlapped on work surface, then matrix multiplication sums energy contents of all pulse overlapped areas based on how many pulses share that area simultaneously.

All six matrices that constitute the mathematical model share one very important common property - each matrix consists of spreadsheet processor's cells. All the cells within the spreadsheet processor's worksheet which contains full instance of the mathematical model have square shape and the same area. This property allows one to refer to each cell as a unit area used throughout current instance of the mathematical model. Each such unit area has four sides of equal unit length.

The ability to refer to mathematical model cells by their common unit area and unit length allows for the model user to measure processes and states within input and output matrices using any preferred arbitrary base unit of length. Because all data within model matrices are of a numeric type, user can select multiple adjacent cells within any of six matrices to see the sum, average, minimum, maximum, and other statistical values representing user selection with the help of spreadsheet processor's standard features.

The sum of numeric content of all cells within input matrix "P" represents the total amount of user-defined or the model's default laser pulse energy which as well can be expressed by user in any preferred base units. By using standard spreadsheet processor function for counting all non-zero cells within matrix "P" one can quickly find the total area of laser pulse planar projection on work surface. By selecting any individual cell of output matrix "YPX" one can view the total amount of energy accumulated by the corresponding unit area of work surface.

One can assume that there will always be potential for finding more new ways of extracting additional information about the distribution of laser pulse planar energy densities. The present method allows its users not only to model the distribution of laser pulse planar energy densities for both laser system types - pulsed and CW but also to combine several different instances of mathematical model by summing all the necessary output matrices "YPX" throughout these open instances and outputting the sum into a new spreadsheet processor's worksheet. Such technique would allow the model user to render each consecutive step of laser pulse delivery to the work surface so that a complex animation can be produced. One can even model processes that are not so easily achievable with common laser systems and their technical parameters.

The user of the mathematical model would often view output matrices zoomed out because of their size thus all numeric data in all the matrices are colour-scaled using spreadsheet processor's standard conditional formatting feature so that numbers are visually represented as colours depending on their relative magnitudes within their corresponding matrices.

\section{Conclusions}

The user's overall understanding of how the present method's mathematical model of matrix multiplication works can help the user extract even more useful information out of it. Therefore method can be used for interactive teaching purposes or to assist advanced users. Finally, the method can help its users better to interpret and to test common lasing formulas as well as to produce new ones, and no similar modelling method, which allows that, yet exists.

During testing of the mathematical model it was found that the better the distribution of planar energy densities within pulse model is defined the more accurate results are produced on the output. The same applies to the resolution, as the smaller unit areas produce better results.

Before the final model was developed, its previous version was implemented using HTML5 Canvas and JavaScript technologies, and pulse overlapping was achieved by visually combining semi-transparent circles, though output results did not provide any numeric data. Therefore final model is based on numeric data, and visualizations are model's by-products. Nevertheless these visualizations can help one spot many important patterns, such as recurring rectangular patterns of length equal to distance between pulses and width equal to distance between lines within output matrix of flat work surface.

\section{Acknowledgements}

This research was supported by laser system equipment from Rofin-Sinar Laser $\mathrm{GmbH}$.

\section{References}

A. J. Antonczak, B. Stepak, P. E. Kozioł, and K. M. Abramski. The influence of process parameters on the laser-induced coloring of titanium. Applied Physics A, 115(3):1003-1013, 2014. doi: 10.1007/s00339-013-7932-8

J. Bliedtner, H. Muller, and A. Barz. Lasermaterialbearbeitung. Fachbuchverlag Leipzig im Carl Hanser Verlag, 2013. doi: 10.3139/9783446429291

J. Eichler and H. J. Eichler. Laser. Springer-Verlag Berlin, 1998. doi: 10.1007/978-3-662-08247-8

P. Laakso, S. Ruotsalainen, H. Leinonen, A. Helle, R. Penttilä, A. Lehmuskero, and J. Hiltunen. Direct color marking of metals with fiber lasers. VTT, Lappeenranta, Finland, 2009. Research Report VTT-R-02403-09

L. Ming, A. Tse, and T. Hoult. Colour marking of metals with fibre lasers. In Proceedings of the 3rd Pacific International Conference on Application of Lasers and Optics, 2008.

V. Veiko, G. Odintsova, E. Ageev, Y. Karlagina, A. Loginov, A. Skuratova, and E. Gorbunova. Controlled oxide films formation by nanosecond laser pulses for color marking. Optics Express, 22(20):24342-24347, 2014. doi: 10.1364/oe.22.024342 